\title{
Interactive Ray Tracing of Arbitrary Implicits with SIMD Interval Arithmetic
}

\author{
Aaron Knoll* \\ SCI Institute, University of Utah \& IRTG \\ Younis Hijazi ${ }^{\dagger}$ \\ Charles Hansen \\ Ingo Wald ${ }^{\S}$ \\ University of Kaiserslautern \& IRTG \\ SCI Institute, University of Utah \\ Hans Hagen ${ }^{\text {II }}$ \\ Intel Corporation \& SCI Institute, University of Utah \\ University of Kaiserslautern
}

\begin{abstract}
We present a practical and efficient algorithm for interactively ray tracing arbitrary implicit surfaces. We use interval arithmetic (IA) both for robust root computation and guaranteed detection of topological features. In conjunction with ray tracing, this allows for rendering literally any programmable implicit function simply from its definition. Our method requires neither special hardware, nor preprocessing or storage of any data structure. Efficiency is achieved through SIMD optimization of both the interval arithmetic computation and coherent ray traversal algorithm, delivering interactive results even for complex implicit functions.
\end{abstract}

Index Terms: I.3.5 [Computer Graphics]: Computational Geometry and Object Modeling - Curve, surface, solid, and object representations; I.3.7 [Computer Graphics]: Three-Dimensional Graphics and Realism-Raytracing

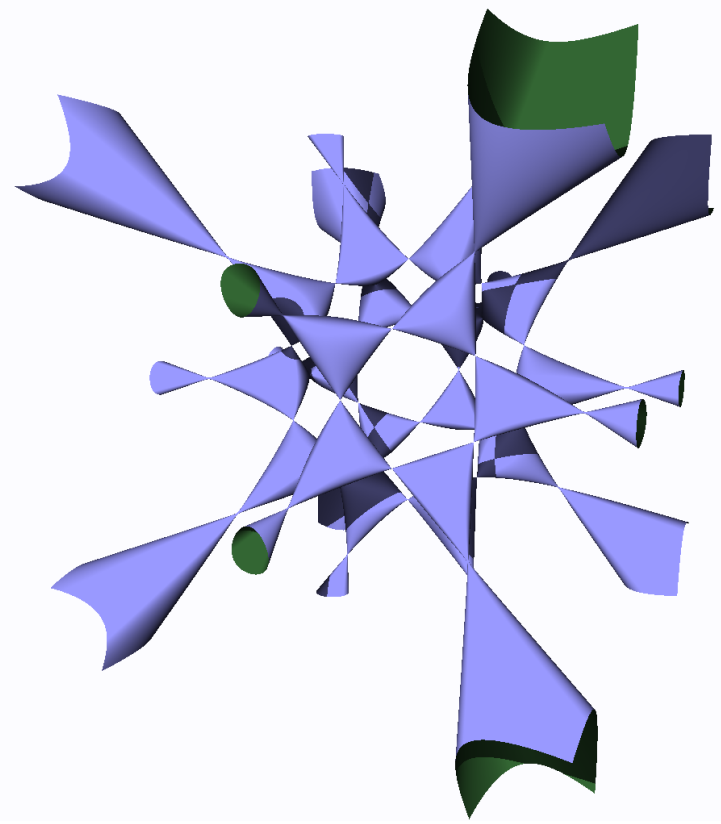

Figure 1: The Barth-sextic Implicit rendered roughly interactively at $9.0 \mathrm{fps}$ (6.1 fps with shadows) with a $512^{2}$ frame buffer on an Intel Core Duo $2.16 \mathrm{GHz}$, purely on the CPU.

*email:knolla@sci.utah.edu

†hijazi@informatik.uni-kl.de

†hansen@sci.utah.edu

\$wald@sci.utah.edu

IIhagen@informatik.uni-kl.de

\section{INTRODUCTION}

In graphics, geometry is most often modeled explicitly as a piecewise-linear mesh. An alternative is a higher-order analytical representation in implicit or parametric form. This option presents advantages, such as compact storage and view-independent local smoothness. While implicits have not experienced as widespread adoption as parametric surfaces in 3D modeling, they are common in other fields, such as mathematics, physics and biology. Moreover, they serve as geometric primitives for isosurface visualization of point sets and volume data.

To render implicits in $3 \mathrm{D}$, one is principally given a choice of extracting and rasterizing a mesh, or ray tracing the surface directly via root-solving. Mesh extraction methods that adaptively reconstruct geometric or topological features exist; however they remain limited in the features they can reproduce, and are not sufficiently fast for dynamic extraction alongside real-time rasterization. While ray tracing low-order implicits is often trivial, arbitrary implicits pose a difficult problem. In the past two decades, several techniques have been developed to ray trace general implicits robustly. Overall, these methods either are slow, restrict the class of functions they handle, or resort to piecewise approximations. Methods involving interval arithmetic (IA) are the most general in that they can accomodate any programmable function. As implemented, however, they are among the least efficient.

Recently, coherent traversal techniques, SIMD vector instructions and multicore CPUs have enabled interactive ray tracing. Applications have largely sought to compete with rasterization in rendering explicit geometries - principally offering scalability to large data, and more powerful, flexible and intuitive shading and lighting models. As geometries that cannot be trivially rasterized, arbitrary implicits make a particularly intriguing application for ray tracing. Coherent ray tracing has not been applied to this problem before, and conventional ray tracing methods are slow largely due to the high computational cost of interval evaluation. By optimizing interval arithmetic with SSE, and pairing this with a fast coherent traversal algorithm, we find that interactive performance is possible on common laptop hardware, with a system that accurately visualizes any implicit surface composable by interval algebra.

The contribution of our work is the combination of a SIMD interval arithmetic library with a novel coherent ray tracing algorithm for implicits that performs coherent spatial bisection without the need for an explicit acceleration structure. We require no special hardware, other than SIMD vector instructions prevalent on all modern CPUs. To render, we require only the implicit function itself, a desired graphing domain, and an appropriate precision criterion or tolerance. We demonstrate our method on various implicits, including difficult cases for extraction-based methods, such as functions with singularities and time-variant 4D hyper-surfaces.

\section{Related WORK}

\subsection{Mesh Extraction}

Application of marching cubes [13, 28] on implicits can generate meshes interactively, but will entirely omit features smaller than the static cell width. Paiva et al. [19] detailed a robust algorithm based on dual marching cubes, using interval arithmetic for visibility in 
conjunction with topological and geometric oracles. Varadhan et al. [25] employed dual contouring and IA to decompose the implicit into patches, and compute a homeomorphic triangulation for each patch. Schreiner et al. [23] used a moving least-square guidance field to adaptively triangulate implicits. Though they generate nice meshes that preserve topology within geometric constraints, these methods are restricted to continuous or compact manifold implicits, and compute offline in the order of seconds or minutes.

\subsection{Ray Tracing Implicits}

The blobby surfaces of Blinn [1] provided modeling interest in an efficient method of rendering implicits. Kalra \& Barr [10] devised a class of L-G surfaces, which could be robustly isolated within a bounding region given a known Lipschitz-condition bound. Stolte \& Caubet [24] applied discrete ray tracing to voxelized representations of implicits. Hart [8] proposed evaluating signed distance functions along a ray, considering balls of diminishing radii separating the ray and a surface. Loop \& Blinn [12] implemented a fast ray casting technique for the GPU that decomposes implicits into piecewise Bézier tetrahedra. Romeiro et al. [21] proposed a hybrid GPU/CPU technique for ray-casting CSG trees of implicits.

\subsection{Ray Tracing Implicits with Interval Arithmetic}

Mitchell [15] was the first to employ interval arithmetic for implicit ray tracing. He devised a hybrid algorithm that employed bisection to segment the ray into intervals on which the function is monotonic, followed by root refinement via a standard numerical root-finding method. Capriani et al. [2] combined interval bisection with various other iterative schemes, including the Interval Newton method. De Cusatis Junior et al. [3] used affine arithmetic, a higherorder interval algebra, to address the bound overestimation problem of pure interval arithmetic (see Section 3.2). Sanjuan-Estrada et al. [22] compared performance of two hybrid interval methods with implementations of the Interval Newton and a recursive pointsampling subdivision method in the POV-Ray framework. Florez et al. [5] proposed a ray tracer that antialiases surfaces by adaptive sampling during interval subdivision. Even when accounting generously for Moore's Law, none of these methods would perform interactively on a modern PC if implemented naïvely.

(a)

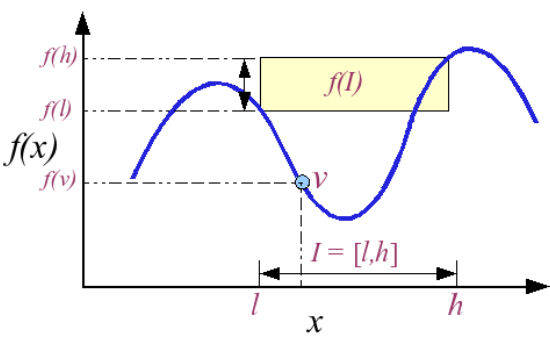

(b)

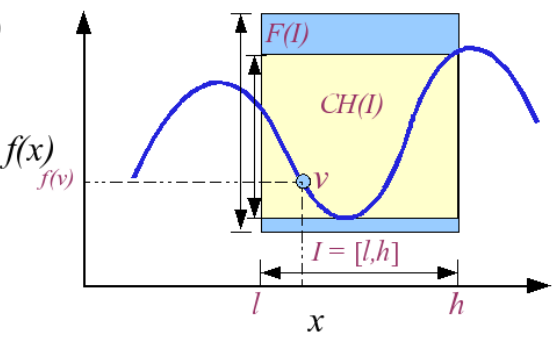

Figure 2: Inclusion property of interval arithmetic. (a) When a function is non-monotonic, simply evaluating the lower and upper components of a domain interval is insufficient to guarantee a convex hull over the range. This is not the case with interval arithmetic (b), which, when evaluated, will encompass all minima and maxima of the function within that interval. Thus, an IA representation $F$ of a function $f$ can definitively determine if $f$ possibly passes through $v$ on an interval $I$, by testing if $v \in F(I)$. Ideally, $F(I)$ is equal or close to the bounds of the convex hull, $\mathrm{CH}(\mathrm{I})$.

\section{BACKGROUND}

\subsection{Implicit Functions}

An implicit surface $S$ in 3D is defined as the set of solutions of an equation

$$
f(x, y, z)=0
$$

where $f: \Omega \subseteq \mathbb{R}^{3} \rightarrow \mathbb{R}$. For our purposes, assume this function is defined by any analytical expression. In ray tracing, we seek the intersection of a ray

$$
\vec{P}(t)=\vec{O}+t \vec{D}
$$

with this surface $S$. By simple substitution of these position coordinates, we derive a unidimensional expression

$$
f_{t}(t)=f\left(O_{x}+t D_{x}, O_{y}+t D_{y}, O_{z}+t D_{z}\right)
$$

and solve where $f_{t}(t)=0$ for the smallest $t>0$.

In this sense, ray tracing is a root-finding problem. For simple implicits such as a plane or sphere, $f_{t}=0$ can be solved for $t$ trivially. More complicated expressions, such as nonalgebraics and polynomials of degree 5 or higher, cannot be solved analytically. Global iterative root-finding methods such as regula falsi can solve over an interval on which a root is known to exist, but fail otherwise. Recursive examinination of sign changes, in conjunction with evaluation, work only when a function is monotonic over an interval. Such "point-sampling" methods (e.g. Kalra \& Barr [10]) succeed when monotonicty assumptions can be made; otherwise they may fail to robustly determine zeros of the implicit, as illustrated in Figure 2(a). Fortunately, interval arithmetic provides us with a mechanism for testing whether or not a zero of a function exists over a sub-domain of the implicit.

\subsection{Interval Arithmetic}

Interval arithmetic was introduced by R. E. Moore [16] as an approach to bounding numerical rounding errors in mathematical computation. The same way classical arithmetic operates on real numbers, interval arithmetic defines a set of operations on intervals. Let $X=[a, b]$ and $Y=[c, d]$ be intervals. Then, if $o p \in\{+,-, *, /\}$, we define $X$ op $Y=\{x$ op $y$ where $x \in X$ and $y \in Y\}$. For example,

$$
\begin{gathered}
X+Y=[a, b]+[c, d]=[a+c, b+d] \\
X-Y=[a, b]-[c, d]=[a-d, b-c] \\
X \times Y=[\min (a c, a d, b c, b d), \max (a c, a d, b c, b d)]
\end{gathered}
$$

Moore's fundamental theorem of interval arithmetic [16] states that for any function $f: \Omega \subseteq \mathbb{R}^{3} \rightarrow \mathbb{R}$ (where $\Omega$ is an open subset of $\mathbb{R}^{3}$ ) and a domain box $B=X \times Y \times Z \subseteq \Omega$ the corresponding interval extension $F: B \rightarrow F(B)$ is an inclusion function of $f$, in that

$$
F(B) \supseteq f(B)=\{f(x, y, z) \mid(x, y, z) \in B\}
$$

Thus, by using interval arithmetic to evaluate $F$, we have a very simple and reliable rejection test for the box $B$ not intersecting $S$,

$$
0 \notin F(B) \Rightarrow 0 \notin f(B)
$$

This property can be used in ray tracing for identifying and skipping empty regions of space. Note, however, that although $0 \notin F(B)$ guarantees the absence of a root on an interval $B$, that the converse does not necessarily hold: one can have $0 \in F(B)$ without $B$ intersecting $S$. When $F(B)$ loosely bounds the convex hull, as in Figure 2(b), IA makes for a poor (though still reliable) rejection test. This overestimation problem is a well-known disadvantage, and is fatal to algorithms relying on iterative evaluation of nondiminishing intervals. 
Fortunately, overestimation error is proportional to domain interval width; therefore IA guarantees convergence to the correct solution when interval domains diminish. This is the case in algorithms such as sweeping computation of hierarchially subdivided domains $[4,9]$, and ray tracing algorithms involving recursive interval bisection $[15,2]$. Though the overestimation problem affects the efficiency of these algorithms, recursive IA methods robustly detect the zeros of an implicit, given an adequate termination criterion such as a sufficiently small precision $\varepsilon$ over the domain, or tolerance $\delta$ over the range.

As explained by Mitchell [14], any function can be expressed as an interval extension by considering its disjoint composition of piecewise-monotonic intervals. This includes non-algebraic piecewise or periodic functions such as modulus, and transcendentals such as exponential, logarithm and trigonometric functions [4]. While rigorous definition of the class of IA-expressible functions falls outside the scope of our work, intuitively one can derive an IA extension for any computable function. Once defined, IA operators are composable, allowing for trivial representation of arbitrary functions by their component real-operators. Ill-defined operations (e.g. division by zero, in Section 5.4), may require special-case handling, but are typically consistent with existing numerical solutions for real numbers.

\subsection{Coherent Ray Tracing}

The principal idea of coherent ray tracing is to perform traversal and intersection on groups, or packets, of rays. In this way, the costs associated with ray tracing are amortized over that group. Aggressive coherent methods often compute traversal steps over a bounding frustum of the packet as opposed to individual rays themselves, e.g. [26, 20]. More conservative methods (e.g. [27]) exploit coherence on a smaller scale, specifically when encouraged by hardware. SIMD instruction sets such as SSE perform four floating point operations in parallel, encouraging operations on packets of four rays. While potential gains are more modest, rays with divergent behaviors may still benefit from instruction-level parallelism.

Coherent ray tracing performs best when rays in a packet behave similarly. Ideally, neighboring rays march in lockstep, requiring the fewest total traversal steps to examine a region of space. In the Wald et al. [26] coherent grid traversal (CGT) algorithm, coherent traversal of rectilinear space is accomplished by choosing a major march axis $K$ corresponding to the dominant ray direction, and examining slices of the other dimensions along fixed $K$ intervals. A hierarchical octree extension of CGT was proposed by Knoll et al. [11], and is the major algorithmic inspiration for this work.

\section{Coherent Ray tracing of Implicits With IA}

Our algorithm simplifies the interval bisection method first proposed by Mitchell [15], and employs a variant of coherent octree traversal [11] as opposed to direct bisection of $t$ intervals along the ray. Together, these decisions allow us to perform bisection in a non-recursive manner, evaluate intervals quickly using SIMD vector instructions, and avoid unnecessary per-step interval multiplication. The simplicity and efficiency of this algorithm allow it to interactively visualize most implicit functions.

The conventional Mitchell algorithm [15] employs interval bisection to reject empty (rootless) intervals. For each nonempty interval, it then computes the gradient interval, and determines whether $0 \notin F_{t}^{\prime}(T)$, i.e. if the function is monotonic over an interval $T$. When this occurrs, Mitchell resorts to a robust numerical "refinement" method, such as non-IA bisection or regula falsi. Interval Newton methods (e.g. $[2,22]$ ) also compute $F_{t}^{\prime}(T)$ periteration. Gradient interval computation proves expensive. Although previous works suggest these techniques offer improved convergence and efficiency compared to pure bisection, that supposition has been weakly scrutinized. In the context of coherent (a)

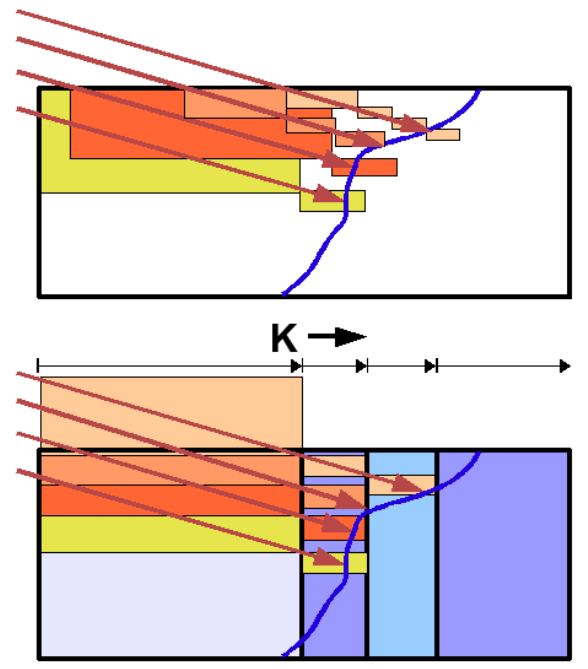

Figure 3: Interval bisection methods. The conventional method (a) recursively bisects each ray along its parameter $t$ until a surface is located to the satisfaction of a termination criterion. Our $K$-marching technique (b) marches rays along a common axis in lockstep. Evaluating along 3D interval boxes $B$ requires slightly less computation per iteration than evaluating the projected function $f_{t}(t)$. More importantly, traversing along a common spatial axis induces more coherent behavior between rays in a packet.

traversal, we find that interval bisection yields unequivocally better performance, and achieves equivalent visual results efficiently at coarser sampling rates.

To leverage SIMD vector operations, we perform interval bisection on four rays at a time. Rather than bisecting $t$ along the ray direction as in Figure 3(a), we bisect space along a major directional axis $K$, similar to the coherent octree volume traversal proposed in [11], and illustrated in Figure 3(b). Particularly when the space between rays exceeds the domain sampling width $\varepsilon$, this ensures more regular sampling of the function across neighboring rays, and preserves the spatial lockstep of coherent traversal (see Section 6.5).

The process of evaluating intervals is then simple. Given an interval box $B=X \times Y \times Z$, our function $f$ and its corresponding IA evaluation $F$, we evaluate whether $0 \in F(B)$ for any ray in the packet. If so, we bisect that interval along the major march axis, or register a hit if a maximum depth threshold is reached. Rather than evaluating the IA extension of the implicit $F_{t}(T)$ projected along the ray, as preferred by previous works, our $K$-bisection method evaluates the 3D implicit $F(X, Y, Z)$ directly. This is convenient as both the IA extension and evaluation functions are natively given as $f(x, y, z)$ expressions. Moreover, our traversal algorithm computes domain intervals $B$ incrementally, requiring only three SSE additions per iteration. Conversely, evaluating $F_{t}(T)$ requires IA evaluation of Equation 3: three IA multiplications and IA additions, or six SSE multiply, min, max and add operations in total.

\section{IMPLEMENTATION}

Our application takes as inputs a domain $\Omega \subseteq \mathbb{R}^{3}$, and an implicit function expression. For simplicity, we chose to hard-code most functions as IA expressions; however the function can also be received from the user as a string and then parsed and compiled into IA code in a dynamic library on-the-fly.

\subsection{SSE Interval Arithmetic}

The foundation of our implicit ray tracing system is our own SSE IA library, which allows us to quickly evaluate intervals in SIMD. Implementation is straightforward; interval multiplication is partic- 


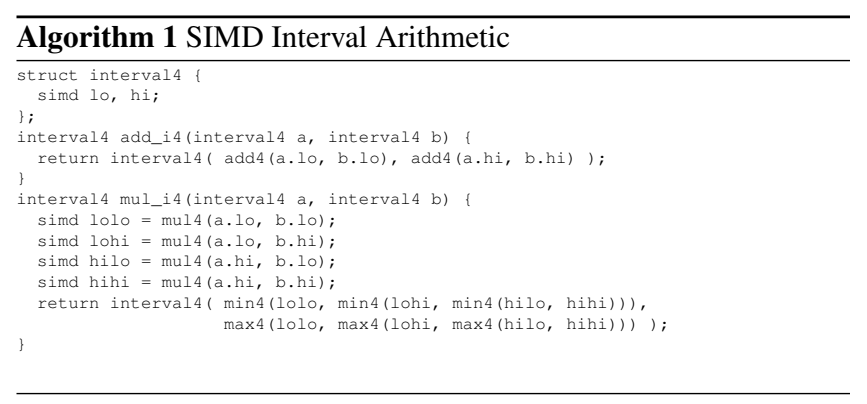

ularly efficient as SSE itself is relatively fast for both multiplication and minimum/maximum operation. The only non-trivial operators are periodic functions such as modulus and sine; and division which requires special-case handling during traversal (see Section 5.4). Examples of SSE IA pseudocode are given in Algorithm 1. We deliberately ignore IA rounding rules for numerical conditioning. For our visualization application, IEEE float rounding errors are insignificant compared to the termination tolerance of our bisection algorithm. One could likely devise numerically ill-conditioned functions that would require IA rounding, but for our purposes it is not a major issue.

\subsection{Ray Packet Structure}

We chose conservative $2 \times 2$ packets for our implementation. Above all, we wish to evaluate baseline performance with SIMD ray tracing using 4-wide SSE vectors; thus behavior of our system should be consistent on wider SIMD hardware, such as a GPU or FGPA. Though larger packets coupled with multi-level algorithms could be significantly faster (e.g. [20]), $2 \times 2$ packet traversal is better-suited for general-purpose ray tracing, and easily allows our implicits to be integrated into a ray tracer as geometric intersection primitives. The actual packet architecture should generalize to any coherent ray tracer; our packet implementation consists of origin and direction stored for each $X, Y, Z$ axis in SSE packed floats. Packets also store the ray hit parameters $t$, and a mask indicating which rays have hit.

\subsection{Traversal}

Once the user has supplied a function, a domain box $\Omega \subseteq \mathbb{R}^{3}$, and a maximum depth $d_{\text {stop }}$, we are ready to perform traversal. As in coherent grid traversal [26], we first find $K$, the dominant axis of the first ray in the packet, and denote the remaining two axes $U$ and $V$. We then perform a standard ray bounding-box test on our domain. We store the actual $t_{\text {enter }}$ and $t_{\text {exit }}$ parameters as well as the intersections with the $K$ entry and exit planes, $t_{\text {Kenter }}$ and $t_{\text {Kexit }}$. Now, we consider the total increment along $K, t_{\text {Kexit }}-t_{\text {Kenter }}$, and compute the total $U$ and $V$ increments over the entire domain. As our implementation is iterative, not recursive, we store an array containing a traversal "stack" for each depth $\left\{0 . . d_{\text {stop }}-1\right\}$, containing the $t$, $K, U$ and $V$ increments bisected at each level.

The algorithm then simply marches from one $K$ slice to the next, incrementing the $t, K, U$ and $V$ positions once per step and keeping track of current and next values, orthogonally for each ray using SSE. It constructs intervals from the $K, U$ and $V$ current and next values. This enables us to iteratively increment domain intervals simply with three SSE additions, as opposed to three SIMD IA multiplications and additions using the conventional $t$-marching method. Branching is only used to omit intervals when $t<t_{\text {enter }}$, and exit when all rays hit successfully or have $t>t_{\text {exit }}$. We store and check a flag for each depth, which indicates when both sides of a $K$-subtree have been traversed. When this happens, we decrement the depth, and exit traversal when depth $=-1$.

At each march iteration, we evaluate the IA function expression on this domain interval $B=X \times Y \times Z$. If $0 \in F(X, Y, Z)$, we "re- curse" by incrementing $d$ and using the bisected increments one level deeper. We register a hit on the surface when $d==d_{\text {stop }}-1$ (or another hit criterion is met, such as $\|F(B)\|<\delta$, as in Section 5.5). Finally, we mask rays that successfully hit or terminate traversal when all rays hit. Traversal is illustrated in Figure 3(b), and pseudocode is given in Appendix A.

\subsection{Division}

IA division requires a slight modification to the above algorithm. In theory, IA division by intervals containing zero is ill-defined, similar to division of real numbers by zero. Fortunately, we can easily detect and handle these cases. For two intervals $A$ and $B$, when $0 \in B$, we define $A / B=[-\infty, \infty]$. When rays traverse these intervals, they will always find a surface within and recurse to maximum depth. Thus, without modification to the traversal, asymptotes will be rendered. To avoid rendering asymptotes, we simply neglect to register a hit when $F_{h i}-F_{l o}=\infty$. This principle is illustrated in Figure 4 . With division correctly handled, our traverser will work for literally any function composed of IA operators.

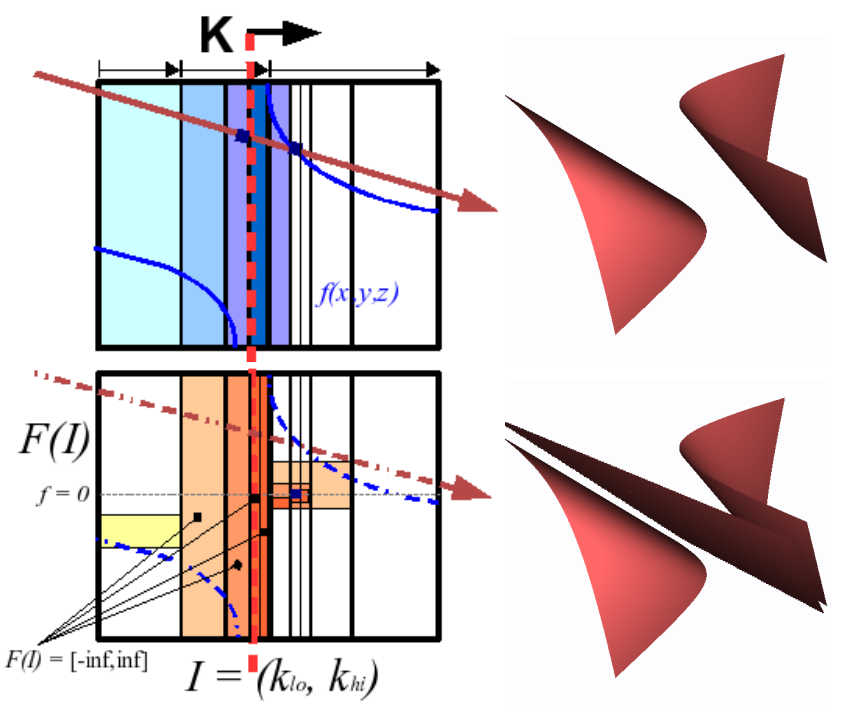

Figure 4: Handling Division. For functions with division, and intervals containing zero near an asymptote, our IA implementation returns "infinite" $F(I)$ intervals (bottom left). As a result, these regions are always subdivided until termination (top left). Fortunately, we may detect this infinite case within the traverser before registering a hit, and thus choose whether or not to visualize asymptotes.

\subsection{Precision Criterion}

In our implementation, $d_{\text {stop }}$ determines the default precision for rendering the implicit. Roughly, this corresponds to a domain precision of $2^{-d_{s t o p}}$, though indeed this varies by ray. However, for a more view-independent domain-space metric, the user may optionally specify an $\varepsilon$, such that $\|B\|_{2}<\varepsilon$ serves as hit criterion, where $B$ is an interval box $X \times Y \times Z$. In this case, the stopping depth is determined adaptively per-packet as

$$
d_{\text {stop }}=\log _{2}\left(\Delta_{\text {packet }} / \varepsilon\right)
$$

where for world-space ray entry and exits $\vec{P}_{r}$ with the domain box $\Omega$, and their corresponding $K$-coordinates $K_{r}$,

$$
\Delta_{\text {packet }}=\max _{r \in \text { packet }} \frac{\left(|| \vec{P}_{r}^{\text {exit }}-\vec{P}_{r}^{\text {enter }}||_{2}\right)^{2}}{\left|K_{r}^{\text {exit }}-K_{r}^{\text {enter }}\right|}
$$

Alternately, the user may specify a range tolerance $\delta$, in which case our algorithm registers a hit when $\|F(B)\|<\delta$. Empirically, the performance differences between these metrics proved minor, and at low precision the $d_{\text {stop }}$ method yields more continuous results for neighboring rays. Thus, we use $d_{\text {stop }}$ as the default metric for evaluating performance at varying sampling quality. 

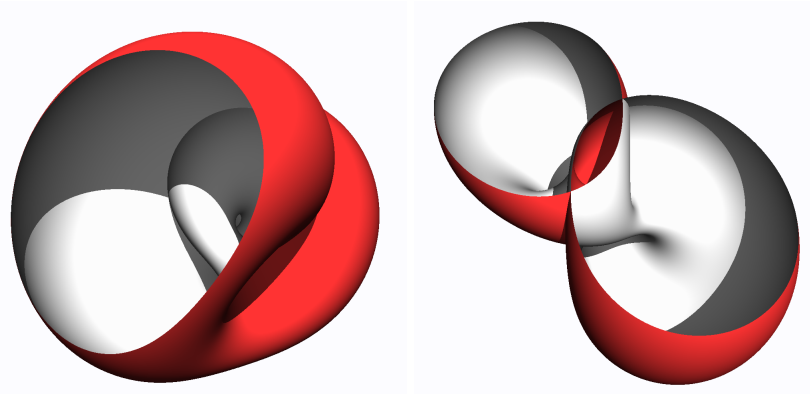

Figure 5: Dynamic shadows aid greately in visualizing the Klein Bottle. Images rendered at $4.0 \mathrm{fps}$ and $2.9 \mathrm{fps}$, respectively at $d_{\text {stop }}=12$.

\subsection{Shadows}

In ray tracing, hard shadows are fairly trivial, requiring a shadow ray cast for every primary camera ray that hits a surface. This typically entails a $20 \%$ to $50 \%$ decrease in frame rate, depending on the coherent behavior of shadow rays. Fortunately, useful shadow rays require less accuracy than primary rays; it frequently suffices to cast shadows to a coarser termination depth, such as $d_{\text {stop }}-2$, while employing a higher depth for primary rays. As shadows are primarily useful as depth cues, this is generally acceptable. The performance penalty is reduced, and loss of shadow detail is seldom perceptible (Figures 1 and 5).
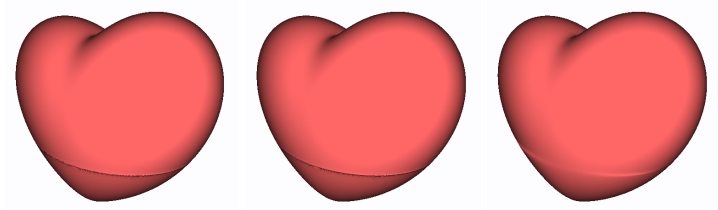

Figure 6: Gradient normal computation, on the Heart function $f(x, y, z)=\left(2 x^{2}+y^{2}+z^{2}-1\right)^{3}-\left(.1 x^{2}+y^{2}\right) z^{3}$. Left: using analytical partial derivatives as gradient, we see shading artifacts where the gradient magnitude approaches zero. Center: with a central differences stencil of width $\Delta_{S}=0.001$, the results are visually indistinguishable. Right: smoother normals with $\Delta_{S}=0.01$. All images render at $6.7 \mathrm{fps}$.

\subsection{Gradient Computation}

For Lambertian shading, we require the surface normal at the ray hit position, given by the $\frac{\partial f}{\partial x}, \frac{\partial f}{\partial y}, \frac{\partial f}{\partial z}$ partial derivatives at that point. While analytical gradients can be manually defined, they are not strictly necessary. If the user fails to define partials, we employ central differences by evaluating our function (using SSE, not SSE IA evaluation) six times to create a central differences stencil. The results look excellent in most cases, and have no appreciable impact on performance. We allow the user to specify stencil width; this is frequently beneficial for surface regions with near-zero gradient magnitude (Figure 6).

\section{Results}

\subsection{General Performance}

All benchmarks were performed on an Intel Core Duo $2.16 \mathrm{GHz}$ laptop with a $512^{2}$ frame buffer. Figure 9 shows various implicit surfaces with their associated equations and performance. Our system achieves well over 20 frames per second for simple objects such as the torus, sphere and conic sections. For more complex objects, performance can fall below interactive speeds on our hardware, but generally exploration at 1-5 fps is possible even for the worst cases. Complicated implicits such as the Barth-sextic exhibit similar performance. Most importantly, we are not restricted to any particular class of surfaces. Non-differentiable, non-continuous, non-manifold, self-intersecting and linked implicits are all robustly rendered.

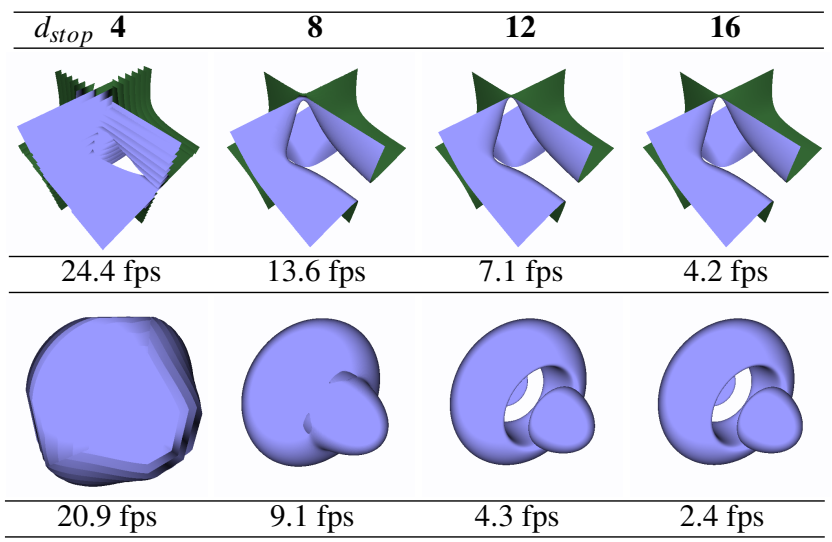

Figure 7: Quality at various $d_{\text {stop }}$ bisection depths. Performance is inversely proportional to depth. Top: the 1st-order Lagrangian trilinear interpolant patch, a cubic implicit, yields tight intervals and converges quickly to the correct contour. Bottom: the Mitchell function causes relatively high IA bound overestimation, and requires greater depth for correct visualization. Even here, a coarse precision criterion $\varepsilon<10^{-3}$ is sufficient to capture the correct topology.

\subsection{Precision and Quality}

We use a common bisection depth $d_{\text {stop }}$ for benchmarking, which corresponds to a domain precision of $2^{-d_{\text {stop }}}$ along the $K$ axis of a given packet. The minimum depth required for accurate visualization depends largely on the bound overestimation of the composed IA rules for that function (Figure 7). As seen in Figure 9, $d_{\text {stop }}=10$ is in practice a good balance of performance and feature reproduction for the vast majority of functions we test. This finding is surprising: a domain precision of $\varepsilon=2^{-10} \approx 10^{-3}$ suffices to accurately visualize most implicits.
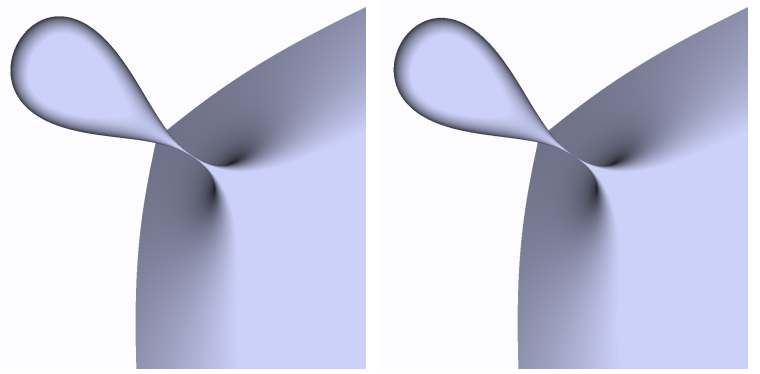

Figure 8: Reproduction of fine features. Though robust for each individual ray, ray tracing (as opposed to beam tracing) may fail to capture infinitely thin features. Coarser-contour visualization at lower precision actually aids in understanding these functions. Left: $d_{\text {stop }}=10$ at $11 \mathrm{fps}$. Right: $d_{\text {stop }}=14$ at $6.5 \mathrm{fps}$.

\subsection{Feature Reproduction}

The tear drop implicit (Figure 8) demonstrates how our algorithm can reproduce fine details that extraction-based approaches often omit. View-independent mesh extraction methods, e.g. [19], frequently fail to capture such regions of a surface, leading to misclassification of details such as asymptotes, singularities or infinitely thin connected surfaces. However, when thin regions or singularities lie between two rays and the interval bounds are sufficiently small, both discrete rays will (correctly) miss the surface, even though that surface would be encountered by an interval beam. To accurately reproduce such sub-pixel features would be expensive, requiring both supersampling and beam tracing of ray intervals, as detailed by Gavriliu [7]. Rendering at lower precision can actually aid in visualizing these features, as the IA inclusion property guarantees that our rendered surface will always form a convex contour of the actual zero-set (Figure 7). In this way, the user can iteratively modify $d_{\text {stop }}$ until the true surface topology is understood. 

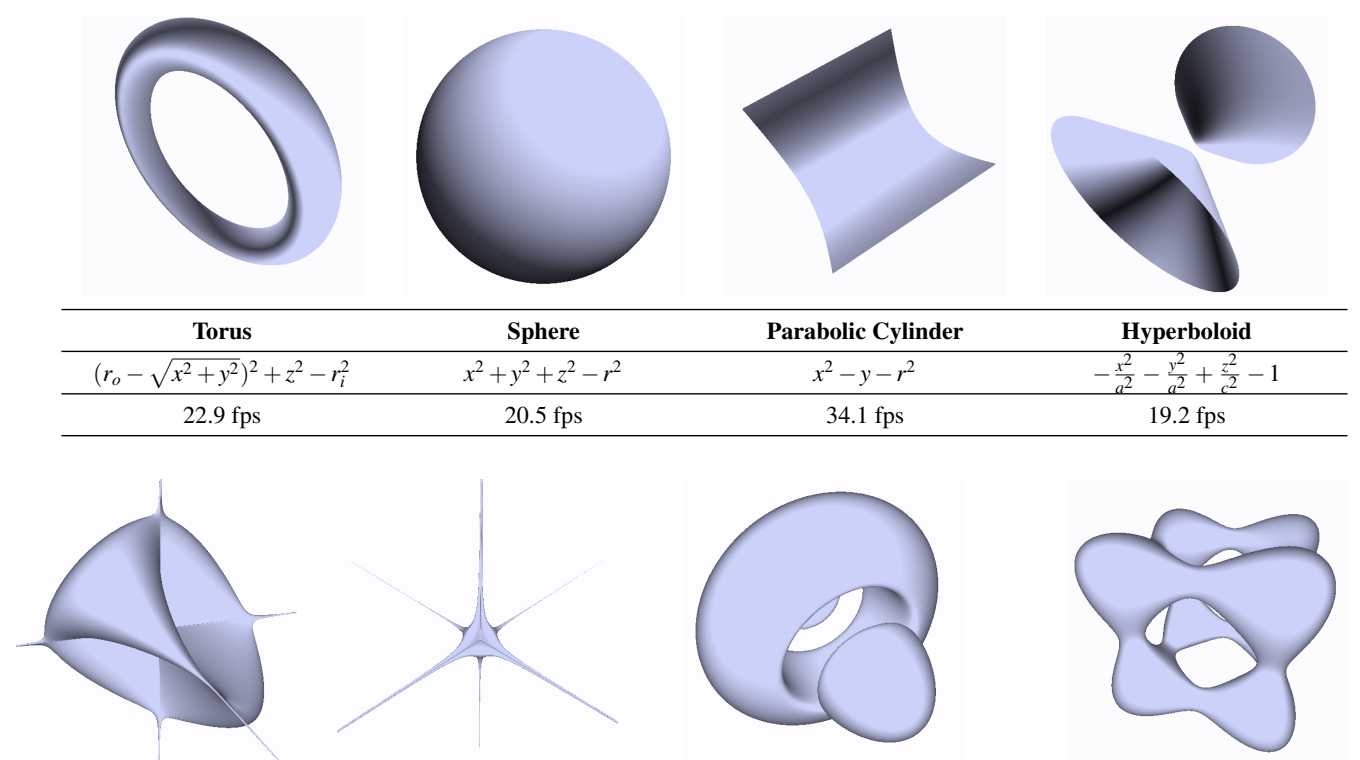

\begin{tabular}{cccc}
\hline Steiner 1 & Steiner 2 & Mitchell & Tangle \\
\hline$x^{2} y^{2}+y^{2} z^{2}+x^{2} z^{2}+x y z$ & $\left(x^{2} y^{2}+y^{2} z^{2}+x^{2} z^{2}\right)^{2}+x y z$ & $4\left(x^{4}+\left(y^{2}+z^{2}\right)^{2}\right)+17 x^{2}\left(y^{2}+z^{2}\right)$ & $x^{4}-5 x^{2}+y^{4}-5 y^{2}+z^{4}-5 z^{2}+11.8$ \\
& & $-20\left(x^{2}+y^{2}+z^{2}\right)+17$ & \\
\hline $6.1 \mathrm{fps}$ & $17.2 \mathrm{fps}$ & $5.9 \mathrm{fps}$ & $3.8 \mathrm{fps}$ \\
\hline
\end{tabular}
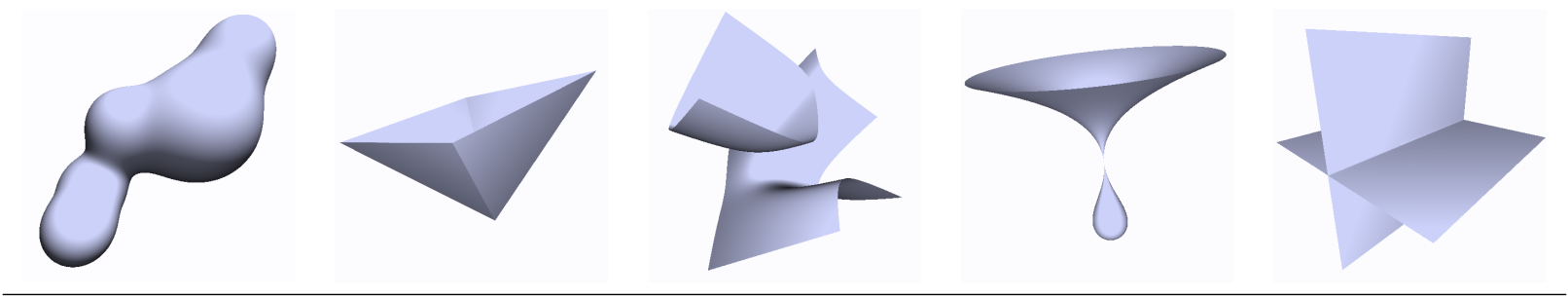

\begin{tabular}{ccccc}
\hline Blobby & Absolute value & Inverse function & Teardrop & Intersecting planes \\
\hline$\sum_{i=1}^{N} \frac{r_{i}^{2}}{\left\|x-p_{i}\right\|^{2}}-1$ & $|x|+|y|-z$ & $\frac{1}{x-y^{2}}-z$ & $0.5 x^{5}+0.5 x^{4}-y^{2}-z^{2}$ & $x y$ \\
\hline $4.7 \mathrm{fps}$ & $30.8 \mathrm{fps}$ & $20.8 \mathrm{fps}$ & $15.3 \mathrm{fps}$ & $33.0 \mathrm{fps}$ \\
\hline
\end{tabular}
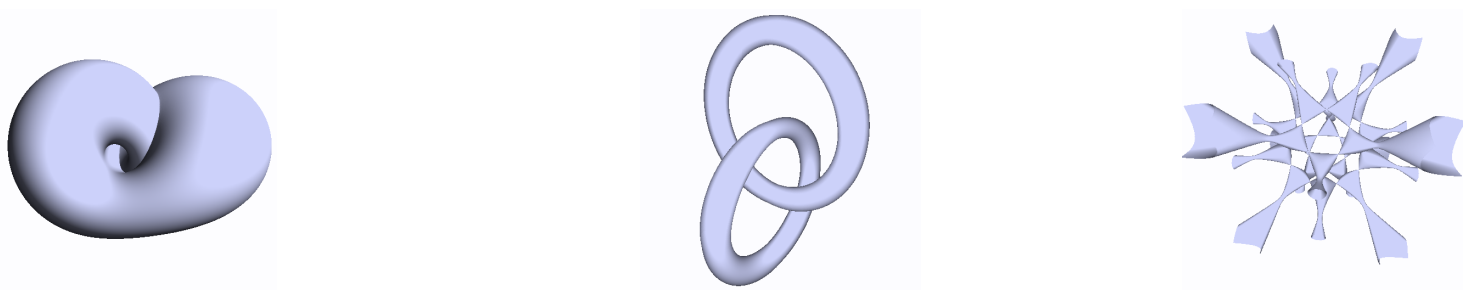

\begin{tabular}{ccc}
\hline Klein Bottle & Linked tori & Barth-sextic \\
\hline$\left(x^{2}+y^{2}+z^{2}+2 y-1\right)\left(\left(x^{2}+y^{2}+z^{2}-2 y-1\right)^{2}-8 z^{2}\right)$ & $g(10 x, 10 y-2,10 z, 13) g(10 z, 10 y+2,10 x, 13)+1000$ & $4\left(\tau^{2} x^{2}-y^{2}\right)\left(\tau^{2} y^{2}-z^{2}\right)\left(\tau^{2} z^{2}-x^{2}\right)$ \\
$+16 x z\left(x^{2}+y^{2}+z^{2}-2 y-1\right)$ & $g(x, y, z, c)=\left(x^{2}+y^{2}+z^{2}+c\right)^{2}-53\left(x^{2}+y^{2}\right)$ & $-(1+2 \tau)\left(x^{2}+y^{2}+z^{2}-1\right)^{2}$ \\
\hline $6.0 \mathrm{fps}$ & $4.0 \mathrm{fps}$ & $4.9 \mathrm{fps}$ \\
\hline
\end{tabular}

Figure 9: Selected implicit functions, covering a wide range of different shapes and topologies. All examples are rendered at $d_{\text {stop }}=10$ at $512^{2}$ frame buffer resolution, on an Intel Core Duo $2.16 \mathrm{GHz}$. Performance is largely dependent on the number of operations required to evaluate the implicit, the entailed cost of computing the associated IA expressions, and the spatial complexity (effectively, implicit surface area) of the scene. Barth-sextic was rendered using $\tau=\frac{1+\sqrt{5}}{2}$. 


\subsection{Dynamic Scenes}

Because we neither precompute an explicit representation of the object, nor a physical acceleration structure in memory, we have great flexibility in rendering dynamically changing $\mathrm{N}$-dimensional implicits. For example, we can render 4D implicits as 3D over time, using a $f(x, y, z, w)$ expression. An example of a two-sheeted hyperboloid morphing into a torus is shown in Figure 10. Though dynamic implicits would be difficult to achieve with mesh extraction techniques, they are trivial in our ray tracing system.

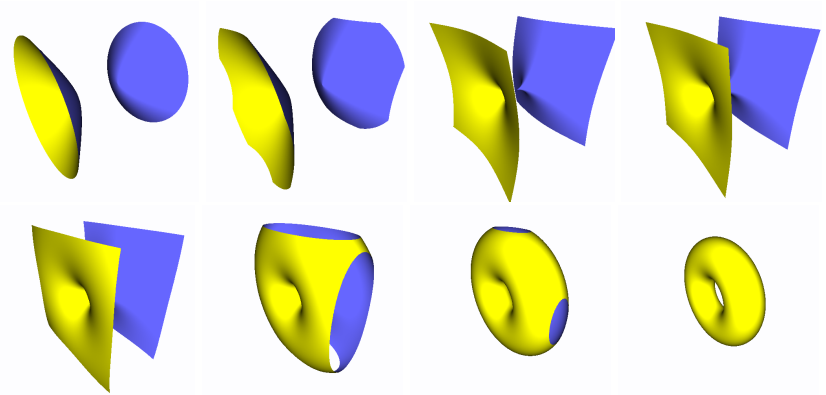

Figure 10: Animated 4D implicits. As our algorithm does not compute or store any acceleration structure, we can make arbitrary changes to the implicit function on the fly. In this example, we interactively morph a hyperboloid into a torus at 9-20 fps.

\subsection{Algorithm Performance Analysis}

Perhaps our most striking finding is that practical IA-based implicit rendering is not inherently slow, even though previous techniques yielded generally poor performance. Implementations such as Mitchell [15] and Capriani et al. [2] sought to render implicits at up to machine precision (up to $\varepsilon=10^{-7}$ ) with superlinearly convergent numerical methods. Despite its slower theoretical convergence, we find that pure interval bisection is more efficient than these methods, particularly at lower precision which is more than adequate for correct visualization (see Section 6.2). To verify this, we implement an SSE variation of the Mitchell [15] algorithm, which performs interval bisection until all rays in the packet have $0 \notin F^{\prime}(B)$, followed by non-interval bisection for root refinement. Implemented in SSE, this method proves far slower than pure bisection, even with small $\varepsilon$. In addition, we compare our $K$-marching algorithm with a standard $t$-bisection. For large, partical $\varepsilon$, standard $t$-marching only performs $5 \%-20 \%$ slower, depending on scene and computational demand of implicit evaluation. However, at smaller $\varepsilon$, where the actual domain intervals of neighboring rays diverge spatially (Figure 3(a)), coherence suffers and $K$-marching is significantly more efficient, potentially by an order of magnitude. These findings are summarized in Table 1, and overall encourage implementation of our $K$-marching method.

\begin{tabular}{|l|c|c|c|c|c|c|}
\hline Algorithm & \multicolumn{9}{|c|}{$K$-bisect } & \multicolumn{2}{c|}{$t$-bisect } & \multicolumn{2}{c|}{ Mitchell } \\
\hline Domain $\varepsilon$ & $1 \mathrm{e}-3$ & $2 \mathrm{e}-7$ & $1 \mathrm{e}-3$ & $2 \mathrm{e}-7$ & $1 \mathrm{e}-3$ & $2 \mathrm{e}-7$ \\
\hline FUNCTION & \multicolumn{9}{|c|}{ FPS } \\
\hline trilerp & 10.6 & 2.8 & 9.9 & 0.31 & 1.20 & 0.75 \\
\hline mitchell & 5.9 & 1.3 & 5.7 & 1.0 & 0.61 & 0.24 \\
\hline
\end{tabular}

Table 1: Algorithm performance comparison between our $K$ bisection method, an SSE $2 \times 2$ packet implementation of the Mitchell [15] algorithm, and a pure $t$-marching interval bisection. For the $K$-bisection method, these $\varepsilon$ correspond to $d_{\text {stop }}=10$ and $d_{\text {stop }}=22$. Refer to Figure 7 for images of the trilinear interpolant (trilerp) and Mitchell functions.

\subsection{Comparison to Existing Techniques}

It is difficult to assess the performance of comparable works in implicit IA ray tracing. Fortunately, many papers evaluate performance with a sphere. [3] reported around $1.3 \mathrm{fps}$ at $64 \times 64$ on a Pentium 166. Accounting generously for Moore's Law (doubling performance every 18 months), we still achieve between two and three orders of magnitude better performance. Similarly, the hybrid and Interval Newton methods benchmarked in [22] perform at two to three orders of magnitude slower than our method. Florez et al. [5] rendered a sphere in 40 seconds at $300 \times 300$ resolution on a P4 $2.4 \mathrm{GHz}$, albeit with adaptive antialiasing; again our method delivers over two orders of magnitude better frame rate (Figure 9).

\section{CONCLUSION}

We have detailed a coherent ray tracing technique for rendering arbitrary implicit functions. By combining a coherent traversal algorithm with an efficient SSE interval arithmetic library, we are able to visualize implicits robustly, accurately, and interactively at rates over two orders of magnitude faster than previous implementations.

Possibilities for extending our system abound. Performance could be further improved by using larger packets and multilevel coherent ray tracing techniques. Adaptive methods (e.g. [5]) might be desirable for better image quality at lower cost, particularly in conjunction with beam tracing (e.g. [7]), which could robustly antialias thin features and singularities. Performance with computationally difficult implicits, and particularly those with high bound overestimation, would improve with a higher-order inclusion rule set such as affine arithmetic [3] or midpoint-Taylor arithmetic [7]. Though it would entail some sacrifice in generality and portability, a similar interval bisection algorithm would be simple to implement, and likely fast, as a fragment program on the GPU.

While powerful, our method has some limitations. It is not an interval beam tracer; aliasing may occur when rendering functions with sub-pixel features at small tolerance. Though interactive for most implicits we tested, it is still computationally demanding and may not be as fast as special-case intersections, particularly for lower order implicits. More generally, implicits have not experienced widespread adoption in graphics compared to explicit modeling methods for smooth surfaces such as subdivision surfaces, though this has perhaps been partly due to their difficult rendering.

An immediate application for this work is a general-purpose 3D graphing application, for use in conjunction with a mathematical software package. CPU ray tracing is particularly attractive for this task as it requires no specialized graphics hardware. Ultimately, the ability to efficiently render general implicits could have interesting implications in graphics. Point-set rendering methods such as MPU [18] relying on rational implicits could easily be ray-traced using this technique. Procedural noise implicits could be employed for surfaces, as in [6]. In visualization, isosurfaces of higher-order finite elements [17] could be more efficiently rendered. Also of interest would be using a similar IA technique to ray-trace arbitrary parametric surfaces, as suggested by Mitchell [14].

\section{ACKNOWLEDGMENTS}

This work has been supported by the US Deparment of Energy Center for the Simulation of Accidental Fires and Explosions (CSAFE) grant W-7405-ENG-48; the National Science Foundation, CISE grants CRI-0513212, CCF-0541113 and SEII-0513212; and the Director, Office of Advanced Scientific Computing Research of the U.S. Department of Energy under Contract DE-FE02-06ER25781, the SciDAC Visualization and Analytics Center for Enabling Technologies (VACET). Further support has been provided by the German Science Foundation (DFG IRTG 1131) as part of the International Research Training Group; and through a visiting professorship sponsored by Intel Corp. Special thanks to John C. Hart, Burkhard Lehner, Matthias Gross, Andrew Kensler, Peter Djeu and Warren Hunt for their support and insights.

\section{REFERENCES}

[1] James Blinn. A Generalization of Algebraic Surface Drawing. ACM Transactions on Graphics, 1(3):235-256, July 1982. 
[2] Ole Capriani, Lars Hvidegaard, Mikkel Mortensen, and Thomas Schneider. Robust and efficient ray intersection of implicit surfaces. Reliable Computing, 6:9-21, 2000.

[3] A. de Cusatis Junior, L. de Figueiredo, and M. Gattas. Interval methods for raycasting implicit surfaces with affine arithmetic. In Proceedings of XII SIBGRPHI, pages 1-7, 1999.

[4] Tom Duff. Interval arithmetic and recursive subdivision for implicit functions and constructive solid geometry. In SIGGRAPH '92: Proceedings of the 19th annual conference on Computer graphics and interactive techniques, pages 131-138, New York, NY, USA, 1992. ACM Press.

[5] J. Florez, M. Sbert, M.A. Sainz, and J. Vehi. Improving the interval ray tracing of implicit surfaces. In Lecture Notes in Computer Science, volume 4035, pages 655-664, 2006.

[6] Manuel N. Gamito and Steve C. Maddock. Ray casting implicit fractal surfaces with reduced affine arithmetic. Vis. Comput., 23(3):155-165, 2007

[7] Marcel Gavriliu. Towards More Efficient Interval Analysis: Corner Forms and a Remainder Interval Newton Method. PhD thesis, California Institute of Technology, July 2005.

[8] J. C. Hart. Sphere tracing: A geometric method for the antialiased ray tracing of implicit surfaces. The Visual Computer, 12(10):527-545, 1996.

[9] Y. O. Hijazi and T. M. Breuel. Computing arrangements using subdivision and interval arithmetic. In Proceedings of the Sixth International Conference on Curves and Surfaces Avignon, pages 173-182, 2006.

[10] D. Kalra and A. H. Barr. Guaranteed ray intersections with implicit surfaces. In SIGGRAPH '89: Proceedings of the 16th annual conference on Computer graphics and interactive techniques, pages 297306, New York, NY, USA, 1989. ACM Press.

[11] Aaron Knoll, Charles Hansen, and Ingo Wald. Coherent Multiresolution Isosurface Ray Tracing. Technical Report UUSCI-2007-001, SCI Institute, University of Utah, 2007. (submitted for publication).

[12] Charles Loop and Jim Blinn. Real-time GPU rendering of piecewise algebraic surfaces. In SIGGRAPH '06: ACM SIGGRAPH $2006 \mathrm{~Pa}$ pers, pages 664-670, New York, NY, USA, 2006. ACM Press.

[13] William E. Lorensen and Harvey E. Cline. Marching Cubes: A High Resolution 3D Surface Construction Algorithm. Computer Graphics (Proceedings of ACM SIGGRAPH), 21(4):163-169, 1987.

[14] D. P. Mitchell. Three applications of interval analysis in computer graphics, 1991.

[15] Don Mitchell. Robust ray intersection with interval arithmetic. In Proceedings on Graphics Interface 1990, pages 68-74, 1990.

[16] R. E. Moore. Interval Analysis. Prentice Hall, Englewood Cliffs, NJ, 1966.

[17] Blake Nelson and Robert M. Kirby. Ray-tracing polymorphic multidomain spectral/hp elements for isosurface rendering. IEEE Transactions on Visualization and Computer Graphics, 12(1):114-125, 2006.

[18] Yutaka Ohtake, Alexander Belyaev, Marc Alexa, Greg Turk, and Hans-Peter Seidel. Multi-level partition of unity implicits. ACM Trans. Graph., 22(3):463-470, 2003.

[19] Afonso Paiva, Hlio Lopes, Thomas Lewiner, and Luiz Henrique de Figueiredo. Robust adaptive meshes for implicit surfaces. In 19th Brazilian Symposium on Computer Graphics and Image Processing, pages 205-212, 2006.

[20] Alexander Reshetov, Alexei Soupikov, and Jim Hurley. Multi-Level Ray Tracing Algorithm. ACM Transaction of Graphics, 24(3):11761185,2005 . (Proceedings of ACM SIGGRAPH).

[21] Fabiano Romeiro, Luiz Velho, and Luiz Henrique de Figueiredo. Hardware-assisted Rendering of CSG Models. In SIBGRAPI, pages 139-146, 2006.

[22] J. F. Sanjuan-Estrada, L. G. Casado, and I. Garcia. Reliable algorithms for ray intersection in computer graphics based on interval arithmetic. In XVI Brazilian Symposium on Computer Graphics and Image Processing, 2003. SIBGRAPI 2003., pages 35-42, 2003.

[23] John Schreiner, Carlos Scheidegger, and Claudio Silva. High-Quality Extraction of Isosurfaces from Regular and Irregular Grids. IEEE Transactions on Visualization and Computer Graphics, 12(5):12051212, 2006. Proceedings of IEEE Visualization 2006.

[24] Nilo Stolte and Rene Caubet. Fast High Definition Discrete Ray Trac- ing Implicit Surfaces. In 5th DGCI - Discrete Geometry for Computer Imagery, pages 61-70, Clermont-Ferrand, Universite d'Auvergne., 1995.

[25] Gokul Varadhan, Shankar Krishnan, Liangjun Zhang, and Dinesh Manocha. Reliable implicit surface polyonization using visibility mapping. In Proc. of 4th Symposium on Geometry Processing, 2006.

[26] Ingo Wald, Thiago Ize, Andrew Kensler, Aaron Knoll, and Steven G Parker. Ray Tracing Animated Scenes using Coherent Grid Traversal. ACM Transactions on Graphics, pages 485-493, 2006. (Proceedings of ACM SIGGRAPH).

[27] Ingo Wald, Philipp Slusallek, Carsten Benthin, and Markus Wagner Interactive Rendering with Coherent Ray Tracing. Computer Graphics Forum, 20(3):153-164, 2001. (Proceedings of Eurographics).

[28] Geoff Wyvill, Craig McPheeters, and Brian Wyvill. Data structure for soft objects. The Visual Computer, 2:227-234, 1986.

\section{A Traversal Pseudocode}

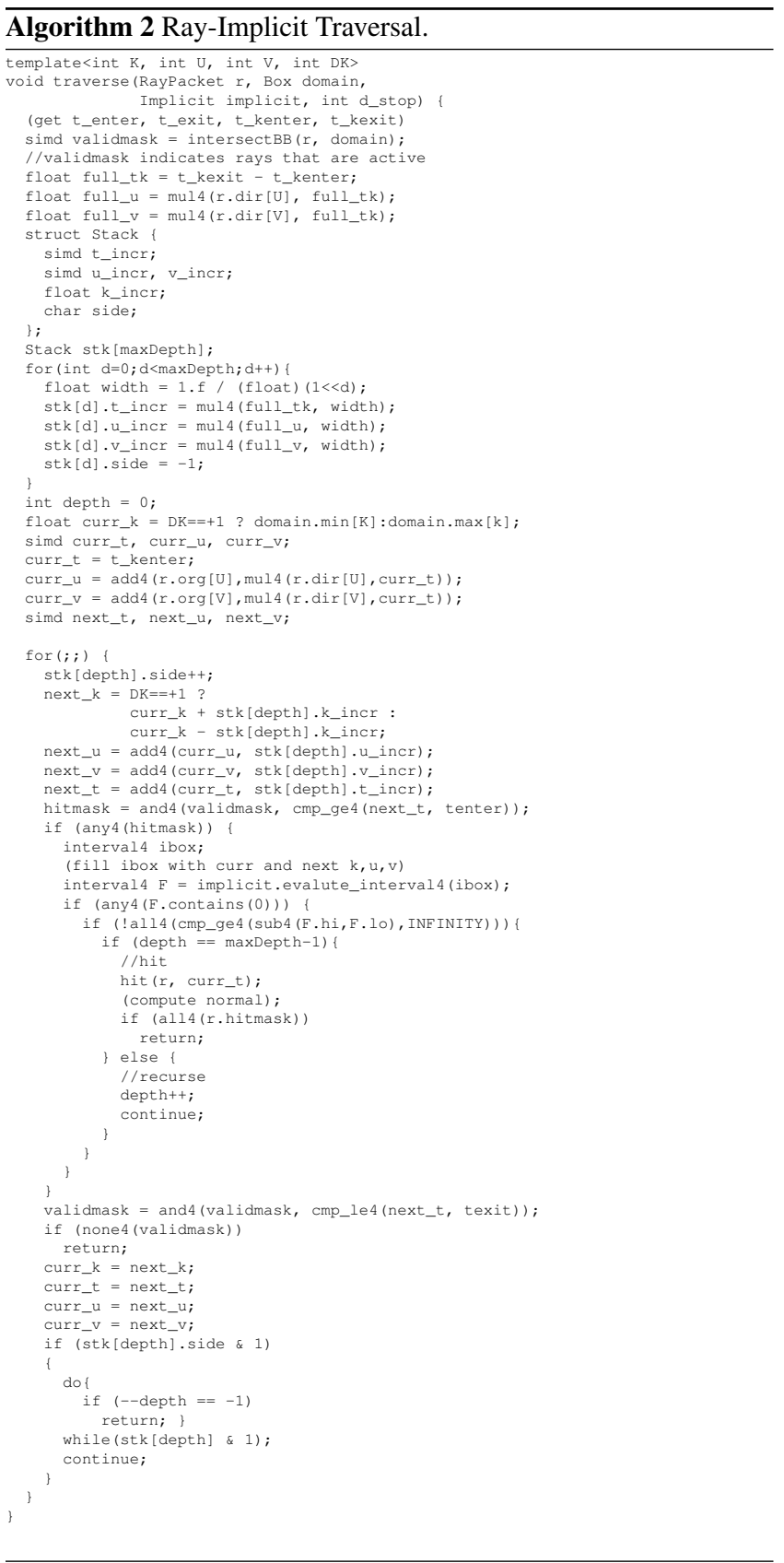

